\title{
Evolution of Elastic Properties of Cold Sprayed Metal Coatings at Elevated Temperatures
}

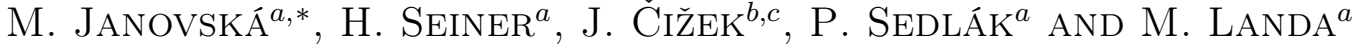 \\ ${ }^{a}$ Institute of Thermomechanics, Academy of Sciences of the Czech Republic, \\ Dolejškova 5, Praha 180 00, Czech Republic \\ ${ }^{b}$ Netme Centre, Institute of Materials Science and Engineering, Brno University of Technology, \\ Technicka 2896/2, 61669 Brno, Czech Republic \\ ${ }^{c}$ Institute of Plasma Physics, Academy of Sciences of the Czech Republic, \\ Za Slovankou 1782/3, 18200 Prague, Czech Republic
}

\begin{abstract}
Resonant ultrasound spectroscopy was applied to analyze the temperature evolution of shear modulus of thick aluminum, nickel, copper and titanium coatings prepared by cold spraying in the temperature range $20^{\circ} \mathrm{C} \rightarrow 560{ }^{\circ} \mathrm{C} \rightarrow 20^{\circ} \mathrm{C}$. The results show that the thermal cycle leads to a significant increase in the shear modulus of nickel, while for copper, titanium and aluminum the shear modulus remains nearly unchanged or exhibits a weak increase only. Evolution of the internal friction parameter shows a significant decrease after the thermal cycle, being the most pronounced for nickel again. The decrease of the internal friction observed in the studied coatings indicates grain coarsening and consequent lower influence of grain boundary sliding.
\end{abstract}

DOI: 10.12693/APhysPolA.134.794

PACS/topics: 62.20.D-, 81.15.Rs

\section{Introduction}

Cold spraying is a promising method for deposition of relatively thick coatings from different types of material [1-3]. Unlike the other thermal spraying methods, the cold spraying process does not involve substantial heating or melting of the sprayed powders, as the formation of layer arises from a severe plastic deformation of the accelerated powder particles upon their impingement at the substrate instead. Materials prepared by cold spraying frequently exhibit superior mechanical and physical properties [1, 4], often comparable to those of the respective bulks. As shown recently by Seiner et al. [5], the cold sprayed metallic coatings exhibit nearly perfect elastic isotropy and high elastic moduli at room temperature. In this paper, we analyze how the elastic moduli (the shear modulus, in particular) of the cold sprayed coatings evolve at elevated temperatures, where the plastically deformed material of the coating is able of recovery and recrystallization. The main aim of this study is to analyze whether the superior elastic performance of the cold-sprayed coatings is retained also at high temperatures and whether the recrystallization processes in these coatings lead to their deterioration or further improvement. Similarly as in [5], the elastic constants of the studied coatings were measured by resonant ultrasound spectroscopy (RUS), which is a modern method capable of accurate contactless determination of the shear elastic moduli.

*corresponding author; e-mail: janovska@it.cas.cz

\section{Materials and experiments}

The examined samples were made from four commercially available powders of dissimilar properties - aluminum, copper, nickel, and titanium. The powder particles possessed different properties such as density (2.67$8.96 \mathrm{~g} \mathrm{~cm}^{-3}$ ), morphology (oval, fully spherical, angular) and average particle diameter $(21-40 \mu \mathrm{m})$. A highpressure cold spray system (PCS-1000, Plasma Giken, Ltd., Japan) was used for the powders deposition onto polycrystalline 6061 aluminum alloy substrates of commercial purity. The minimal volumes of the coating were $150 \times 50 \times 10 \mathrm{~mm}^{3}$. For more details, see [5]. From each of the sprayed materials, small plates of a rectangular parallelepiped-shape (approximately $3.5 \times 2.5 \times 0.5 \mathrm{~mm}^{3}$ ) were cut from the region close to the center of the sprayed area and close to the substrate-coating interface. The largest face of each sample was mirror polished in order to enable scanning by a laser vibrometer. The elastic constants of the examined materials were determined by RUS $[6,7]$. A fully contactless experimental setup described in detail in $[8]$ was used.

The ultrasonic vibrations were excited by an infrared laser pulse focused on the bottom face of the sample and recorded by laser vibrometer on the upper polished face. The experiments were done in the temperature-controlled chamber (accuracy $0.01 \mathrm{~K}$ ) under low pressure nitrogen atmosphere (approximately 10 mbar). The RUS measurements were done in a $20^{\circ} \mathrm{C} \rightarrow 560^{\circ} \mathrm{C} \rightarrow 20^{\circ} \mathrm{C}$ temperature cycle for each sample. The average heating rate was approximately $2.8^{\circ} \mathrm{C} / \mathrm{min}$, including a $90 \mathrm{~s}$ stabilization time at each temperature point. Resonant spectra of the free elastic vibrations of the samples were recorded in a frequency range $100 \mathrm{kHz}-1 \mathrm{MHz}$, which covered between 
10 to 20 from the first 40 resonant frequencies. Based on the results of [5] it was assumed that all coatings exhibit elastic isotropy with only two independent elastic constants, the shear modulus $G$ and the bulk modulus $K$. As the RUS method is dominantly insensitive to the bulk modulus, the shear modulus $G$ was selected as the observed parameter for analysis of the temperature evolution of the elastic properties of the layers. Temperature dependences of the shear modulus $G$ were determined from the evolution of resonant spectra of the samples (including typically $5-10$ resonant frequencies) using the inverse procedure described in detail in [9].

Changes of dimensions of the samples with temperature due to thermal expansion and the corresponding changes in density were taken into account. The linear expansion coefficients of pure aluminum $\alpha=$ $22.2 \mathrm{ppm} / \mathrm{K}$, copper $\alpha=16.6 \mathrm{ppm} / \mathrm{K}$, titanium $\alpha=$ $9 \mathrm{ppm} / \mathrm{K}$ and nickel $\alpha=13 \mathrm{ppm} / \mathrm{K}$ were considered. For nickel, titanium and aluminum, the chosen resonant frequencies were traced throughout the whole temperature cycle. For copper, however, the mirror polished surface become deteriorated at $500{ }^{\circ} \mathrm{C}$ upon cooling, probably by releasing of residual gases from the bulk of the sample (as the measurements were done in a protective low-pressure nitrogen atmosphere). For this reason, the evolution of the shear modulus along the cooling curve was not determinable. At the end of the thermal cycle the copper sample was covered by non-compact black layer, and also a decrease in mass density of the sample from $8.75 \mathrm{~g} / \mathrm{cm}^{3}$ to $8.63 \mathrm{~g} / \mathrm{cm}^{3}$ was observed. After the corrosion products from the surfaces of the $\mathrm{Cu}$ sample were removed, the RUS spectrum at the room temperature was re-measured. At each temperature, the internal friction parameter $Q^{-1}$ was determined from the widths of the dominant resonant peaks in the corresponding resonant spectrum by fitting these peaks by Lorentzian mask [10].

\section{Results and discussion}

Values of shear moduli obtained by isotropic fit from the measured resonant spectra at room temperature are summarized in the first column of Table I. These were used as reference values for determination of temperature evolution of the shear moduli and fall within the range of experimental errors in agreement with anisotropic moduli from [5]. In the second column of Table I, the shear moduli of the coatings after the thermal cycle are shown. It is seen that while for aluminum and titanium the differences of the shear moduli before and after the thermal cycle are small and fully within the experimental error, a very strong stiffening is observed for nickel. Minor stiffening comparable with the experimental error is seen also for copper. However, in this case the above mentioned corrosion of the sample and the accompanying changes in its density must be taken into account.

Temperature dependences of the shear moduli during heating up to $560^{\circ} \mathrm{C}$ and subsequent cooling for all measured samples are shown in Fig. 1. It is clearly seen that
Isotropic shear moduli of cold sprayed layers.

TABLE I

\begin{tabular}{l|c|c}
\hline \hline Material & $\begin{array}{c}G[\mathrm{GPa}] \\
\text { as coated }\end{array}$ & $\begin{array}{c}G[\mathrm{GPa}] \text { after } \\
\text { thermal cycle }\end{array}$ \\
\hline aluminum & $23.2 \pm 2.0$ & $22.8 \pm 2.0$ \\
copper & $42.8 \pm 2.0$ & $44.7 \pm 2.0$ \\
nickel & $57.7 \pm 2.0$ & $66.3 \pm 2.0$ \\
titanium & $34.1 \pm 2.0$ & $35.2 \pm 2.0$
\end{tabular}
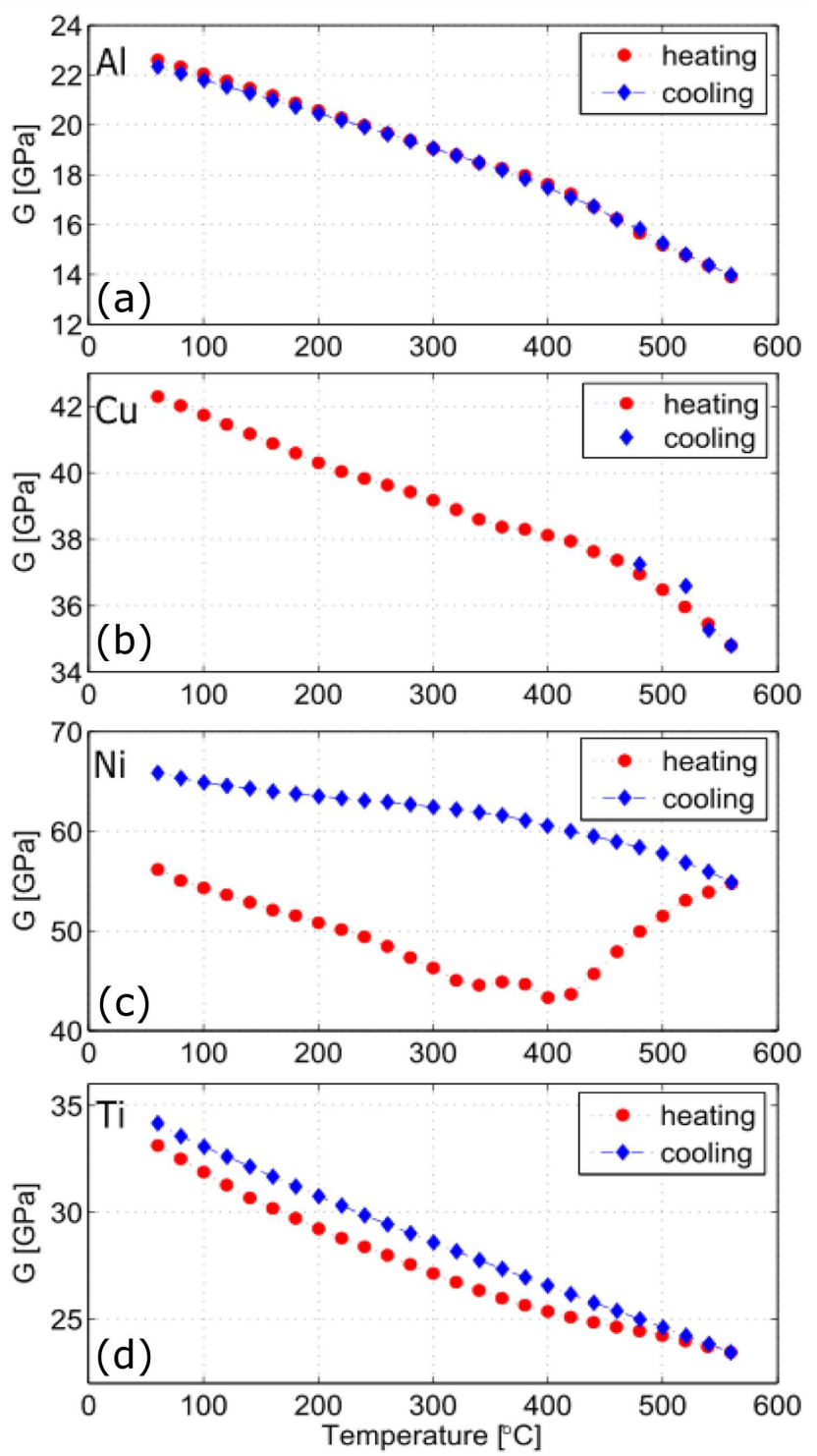

Fig. 1. Temperature evolution of shear modulus $G$ : (a) aluminum, (b) copper, (c) nickel, and (d) titanium.

the particular materials show different evolution of the $G$ modulus during this thermal cycle. Aluminum shows monotonous decrease of the $G$ modulus during the heating and subsequent nearly reversible increase back to the value less than $2 \%$ lower than the initial value. Copper shows similar behavior as aluminum during heating and also in the first part of cooling. As explained in the previous section, the behavior during the cooling phase 



Fig. 2. Temperature evolution of internal friction parameter $Q^{-1}$ : (a) aluminum, (b) copper, (c) nickel, and (d) titanium.

is not clear due to the damage of the mirror polished surface at higher temperatures. The nickel sample exhibited the strongest temperature dependence of the $G$ modulus from all studied samples. In the temperature range from room temperature to $400^{\circ} \mathrm{C}$, the $G$ modulus decreases with two local minima at $340^{\circ} \mathrm{C}$ and $400^{\circ} \mathrm{C}$. In the temperature range $400{ }^{\circ} \mathrm{C}$ up to $560^{\circ} \mathrm{C}$, the $G$ modulus strongly increases. In the cooling part of the temperature cycle, the $G$ modulus increases slightly. The total increase of $G$ modulus after this thermal cycle is approximately $15 \%$. Titanium coating behaves similarly to aluminum and copper samples in the heating part of the thermal cycle. The $G$ modulus of this coating exhibits monotonous decrease during the heating part of the thermal cycle, while the cooling leads to a monotonous increase of elasticity. In this case, the temperature evolu- tion of elasticity is not reversible. The difference between the values of $G$ modulus of $\mathrm{Ti}$ before and after the thermal cycle is approximately $3 \%$.

The temperature evolution of internal friction parameter $Q^{-1}$ was also studied and is shown in Fig. 2. All studied materials show a decrease of the internal friction parameter after the thermal cycle $20^{\circ} \mathrm{C} \rightarrow 560^{\circ} \mathrm{C} \rightarrow 20^{\circ} \mathrm{C}$. The particular temperature dependences of the internal friction parameters differ in shape and also in the absolute values of the internal friction parameter.

A comparison of internal friction parameters before and after the thermal cycle is shown in Table II. Aluminum and copper samples behave in similar way in the heating part of the thermal cycle. From relatively low value of internal friction parameter $\left(Q^{-1}=2.6 \times 10^{-4}\right.$ $\mathrm{Cu}$ and $1.1 \times 10^{-3} \mathrm{Al}$ ) of the as-coated sample, the internal friction parameter increases up to nearly $2.7 \times 10^{-2}$ for copper at temperature $560^{\circ} \mathrm{C}$ and $4.3 \times 10^{-2}$ for aluminum at temperature $480^{\circ} \mathrm{C}$. The copper sample showed an increase in internal friction parameter in the entire temperature range $20^{\circ} \mathrm{C}-560^{\circ} \mathrm{C}$. The temperature dependence of aluminum sample has a flat maximum around $480^{\circ} \mathrm{C}$. This maximum is also clearly visible in the cooling part of the cycle. We can divide the cooling part of the cycle to two regions: nearly reversible part in temperature range $560^{\circ} \mathrm{C}-380^{\circ} \mathrm{C}$ and a linear decrease of the internal friction in the subsequent cooling to room temperature up to value $2.9 \times 10^{-4}$.

The internal friction parameter of nickel coating had the highest value of all studied coatings $Q^{-1}=1.3 \times 10^{-2}$. The temperature dependence shows only a slight decrease in the internal friction parameter in the heating part of the thermal cycle, while the decrease in the cooling part of the cycle is very strong and leads to a decrease of the internal friction parameter to value $5.1 \times 10^{-4}$. The titanium sample shows fast decrease of the internal friction parameter during the heating part of the thermal cycle, from $6.5 \times 10^{-3}$ to $5 \times 10^{-4}$. This decrease of the internal friction parameter continues during the cooling part of the temperature cycle up to $2.1 \times 10^{-4}$. It was not possible to evaluate the cooling part of the thermal cycle for copper sample because of the coverage of the mirror polished surface by the described unreflecting layer. However, from the resonant spectrum measured at the room temperature after the thermal cycle, a slight decrease in the internal friction parameter is clearly seen $\left(Q^{-1}=4 \times 10^{-5}\right)$. Nevertheless, the change is small as the starting value was very small.

TABLE II

Internal friction parameter of cold spayed materials.

\begin{tabular}{l|c|c}
\hline \hline \multirow{2}{*}{ Material } & \multicolumn{2}{|c}{ Internal friction parameter } \\
\cline { 2 - 3 } & As coated sample & $\begin{array}{c}\text { Sample after } \\
\text { thermal cycle }\end{array}$ \\
\hline aluminum & $1.1 \times 10^{-3}$ & $2.9 \times 10^{-4}$ \\
copper & $2.6 \times 10^{-4}$ & $4 \times 10^{-5}$ \\
nickel & $1.3 \times 10^{-2}$ & $5.1 \times 10^{-4}$ \\
titanium & $6.5 \times 10^{-3}$ & $5 \times 10^{-4}$
\end{tabular}


The decrease of the internal friction parameter after the thermal cycle is the lowest for the copper coating, which shows the lowest value of the internal friction parameter in the original coating, yielding it probably the best quality among the studied coatings. In a similar way, the nickel sample, which exhibits the highest value of the internal friction parameter among the original coatings, shows the biggest decrease in the internal friction parameter from all studied samples. As discussed already in [5], the high internal friction in the cold-sprayed nickel and titanium coatings may be attributed to the intensive plastic deformation of the materials during the spraying process and the resulting grain refinement [11] which enables grain boundary sliding. For the nickel sample, our results for a thermal cycle strongly support such interpretation. At elevated temperatures, the plastically deformed structure becomes recovered and recrystallized and the internal friction significantly decreases. However, an interesting difference is observed between nickel and titanium: while for nickel, the decrease of the internal friction is accompanied also by strong stiffening of the elastic moduli, only a very weak stiffening is observed for titanium. In principle, the grain boundary sliding should not affect the elastic moduli [12]. Hence, the finely grained structure in the as sprayed nickel coating should be attributed to some additional effect, for example the delta-E effect arising from the magneto-elastic coupling.

\section{Conclusions}

The evolution of shear modulus and internal friction parameter with temperature in thermal cycle $20^{\circ} \mathrm{C} \rightarrow 560^{\circ} \mathrm{C} \rightarrow 20^{\circ} \mathrm{C}$ was measured for thick metal coatings of aluminum, copper, nickel, and titanium prepared by cold spraying method. It was found that while aluminum, copper, and titanium exhibited nearly reversible evolutions of shear modulus during the thermal cycle, the cold-sprayed nickel exhibited its strong increase. In other words, while the elastic moduli of the as-sprayed coatings were already very close to the respective values for bulk materials, subjecting the coatings to a thermal cycle led either to maintaining of these high elastic performance of the coatings or even to an increase of the moduli. We have also observed a decrease of the internal friction parameter for all studied samples, with the strongest changes observed for nickel and titanium coatings. The interpretation of these changes is that the thermal cycle caused recovery and recrystallization of the coatings, which resulted in a suppression of grain boundary sliding effects.

This interpretation can be also supported by observation of coatings microstructure before and after the thermal cycle, as illustrated in Fig. 3 for Ni coatings. The triggered grain coarsening is clearly visible, with the biggest observed grains reaching up to $4 \mu \mathrm{m}$ in diameter. The different behavior of the nickel sample can be attributed to its magnetism. The grain refinement induced through the severe plastic deformation leads to a random orientation of magnetic domains, which cause strong magneto-elastic coupling. This effect is subsequently suppressed by the observed grain coarsening and ordering of the magnetic domains during the thermal cycle. The different internal friction evolution upon heating of the titanium sample can be caused by its dissimilar crystallographic structure (hcp). An extended discussion on the specific microstructure of an as-sprayed titanium coating is provided in our earlier work in detail [2].
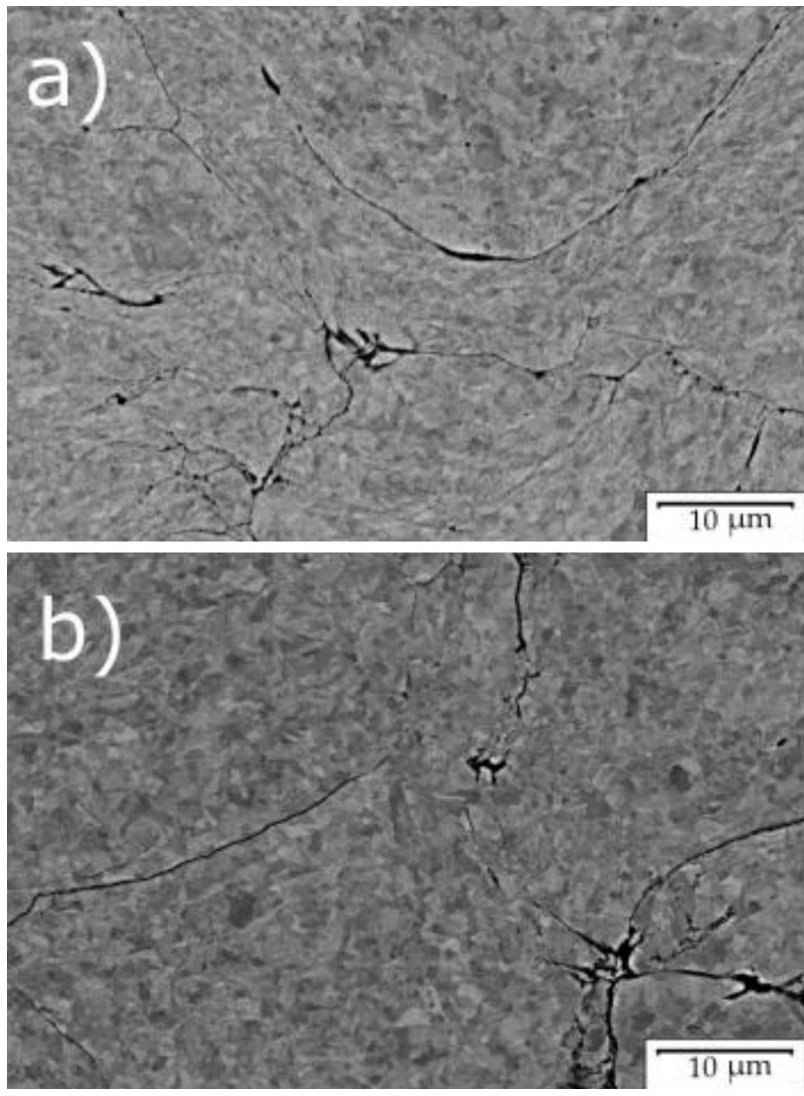

Fig. 3. SEM micrographs of nickel coating: (a) as sprayed, (b) after thermal cycle $20^{\circ} \mathrm{C} \rightarrow 560^{\circ} \mathrm{C} \rightarrow 20^{\circ} \mathrm{C}$.

\section{Acknowledgments}

The work has been financially supported by the Czech Science Foundation project 17-13573S.

\section{References}

[1] A. Papyrin, V. Kosarev, S. Klinkov, A. Alkhimov, V.M. Fomin, Cold Spray Technology, Elsevier, Amsterdam 2007, p. 328.

[2] J. Cizek, O. Kovarik, J. Siegl, K.A. Khor, I. Dlouhy, Surf. Coat. Technol. 217, 23 (2013).

[3] T. Schmidt, H. Assadi, F. Gärtner, H. Richter, T. Stoltenhoff, H. Kreye, T. Klassen, J. Therm. Spray Technol. 18, 794 (2009).

[4] P. Fauchais, J.V.R. Heberlein, M.I. Boulos, Thermal Spray Fundamentals: From Powder to Part, Springer US, New York 2014. 
[5] H. Seiner, J. Cizek, P. Sedlák, R. Huang, J. Cupera, I. Dlouhy, M. Landa, Surf. Coat. Technol. 291, 342 (2016).

[6] A. Migliori, J.L. Sarrao, W.M. Visscher, T.M. Bell, M. Lei, Z. Fisk, R.G. Leisure, Physica B Phys. Condens. Matter 183, 1 (1993).

[7] R.G. Leisure, F.A. Willis, J. Phys. Condens. Matter 9, 6001 (1997).

[8] P. Sedlák, H. Seiner, J. Zídek, M. Janovská, M. Landa, Exp. Mech. 54, 1073 (2014).
[9] M. Landa, P. Sedlák, H. Seiner, L. Heller, L. Bicanová, P. Sittner, V. Novák, Appl. Phys. A Mater. Sci. Process. 96, 557 (2009).

[10] A.S. Nowick, B.S. Berry, Anelastic Relaxation in Crystalline Solids, Academic Press, New York 1972.

[11] Y. Zou, W. Qin, E. Irissou, J.G. Legoux, S. Yue, J.A. Szpunar, Scr. Mater. 61, 899 (2009).

[12] M. Koller, P. Sedlák, H. Seiner, M. Ševčík, M. Landa, J. Stráská, M. Janeček, J. Mater. Sci. 50, 808 (2014). 